\section{Careful observation of hepatic portal venous gas following esophageal variceal band ligation}

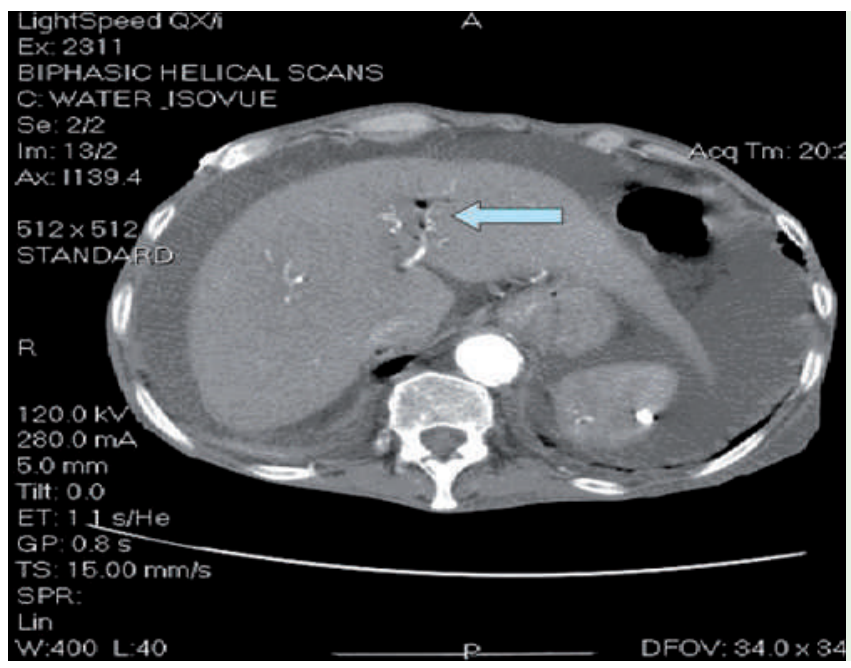

Fig. 1 Portal venous gas seen as an incidental finding on CT scan of the abdomen: gas is present in the left portal vein, superior mesenteric vein, splenic vein, and the confluence of the portal vein.

Hepatic portal venous gas (PVG) is a rare and often puzzling clinical entity, with widely varying features regarding presentation [1].

A 77-year-old white woman with a history of primary biliary cirrhosis presented complaining of hematemesis and underwent an esophagogastroduodenoscopy and band ligation. Three days later, CT of her abdomen was performed and showed the presence of gas in the portal venous system ( Fig. 1) without any evidence of pneumatosis, ischemia, or bowel thickening. The Department of Surgery was consulted for further evaluation of the PVG, but given the patient's debilitated condition and the benign abdominal exam findings, it was elected to continue to monitor her. She did very well, without any complaints of abdominal pain or fever, and was eventually discharged home in a satisfactory condition.

crotizing enterocolitis, PVG has been reCT scanning in the diagnosis and treatthe finding of PVG has been demonstrattis and Crohn's disease [4] gastroduodenoscopy may have been bowel necrosis.
K. Ahmed, M. Atiq, E. Richer, G. Neff, N. Kemmer, K. Safdar

Division of Gastroenterology and Hepatology, Department of Internal Medicine, University of Cincinnati, Cincinnati, Ohio, USA

\section{References}

1 Yamamuro M, Ponsky JL. Hepatic portal venous gas: report of a case. Surg Today 2000; 30: 647-650

2 Wolfe JN, Evans WA. Gas in the portal veins of the liver in infants. Am J Roentgenol Radium Ther Nucl Med 1955; 74: 486-488

3 Kinoshita H, Shinozaki M, Tanimura $\mathrm{H}$ et al. Clinical features and management of hepatic portal venous gas. Arch Surg 2001; 136: $1410-1414$

4 Hou SK, Chern CH, How CKet al. Hepatic portal venous gas: clinical significance of computed tomography findings. Am J Emerg Med 2004; 22: 214-218

5 Schulze CG, Lee SC, Hong DH et al. Portal and superior mesenteric venous gas with retroperitoneal abscess. J Korean Med Sci 1992; 7: $62-65$

\section{Bibliography}

Dol $10.1055 / \mathrm{s}-2007-966850$

Endoscopy 2008; 40: E103

(c) Georg Thieme Verlag KG Stuttgart · New York . ISSN 0013-726X garded as a sign of an abdominal emergency requiring urgent exploratory surgery [2]. With the ever-expanding role of ment of abdominal complaints, however, ed in a wider array of abdominal conditions ranging from bowel necrosis to chronic conditions such as ulcerative coli-

It is not entirely clear what caused the PVG in our patient. In the absence of any abdominal findings and with a normal serum lactate level, an abdominal emergency is less likely. In theory, the esophagoresponsible for causing vascular breach, resulting in PVG without any evidence of

The patient in this case was at high risk for perioperative mortality with underlying cirrhosis, and it was determined that surgical intervention would be more harmful than beneficial.

In conclusion, in selected patients in whom PVG has been demonstrated on imaging, such as the one described here, supportive care may often be all that is required.

Endoscopy_UCTN_Code_CPL_1AH_2AC

\section{Corresponding author}

\section{K. Safdar, MD}

Division of Gastroenterology and Hepatology Department of Internal Medicine

University of Cincinnati

231 Albert Sabin Way

Cincinnati

$\mathrm{OH}$ 45267-0595

USA

Fax: +1-513-558-1744

ksafdar90@yahoo.com 